\title{
Evidence for methane escape and strong seasonal and dynamical perturbations of Neptune's atmospheric temperatures
}

\author{
G. S. Orton ${ }^{1}$, T. Encrenaz ${ }^{2}$, C. Leyrat $^{3}$, R. Puetter ${ }^{4}$, and A. J. Friedson ${ }^{1}$ \\ 1 MS 169-237, Jet Propulsion Laboratory, California Institute of Technology, 4800 Oak Grove Drive, Pasadena, CA 91109, USA \\ 2 LESIA, Observatoire de Paris, CNRS, UPMC, Univ. Paris 7, 5 place Jules Janssen, 91925 Meudon Cedex, France \\ e-mail: Therese.Encrenaz@obspm.fr \\ 3 MS 230-205, Jet Propulsion Laboratory, California Institute of Technology, 4800 Oak Grove Drive, Pasadena, CA 91109, USA \\ 4 Center for Astrophysics \& Space Sciences, CASS 0424, University of California, San Diego, 9500 Gillman Dr., La Jolla, CA, \\ 92093-0424, USA
}

Received 13 July 2007 / Accepted 7 August 2007

\section{ABSTRACT}

\begin{abstract}
Aims. We studied the distribution of mid-infrared thermal emission from Neptune to determine the spatial variability of temperatures and the distribution of trace constituents, allowing us to determine the relative strengths of radiation and dynamics in its atmosphere. Methods. Mid-infrared images of the planet were taken at the Very Large Telescope on 1-2 September 2006.

Results. These images reveal strong inhomogeneities in thermal emission. 17.6 and $18.7-\mu \mathrm{m}$ images exhibit strong seasonally elevated south polar temperatures near Neptune's tropopause. These high temperatures allow tropospheric methane, elsewhere cold-trapped at depth, to escape into the stratosphere. Poleward of $70^{\circ} \mathrm{S}, 8.6-$ and $12.3-\mu \mathrm{m}$ emission from stratospheric methane and ethane is enhanced, and a distinct, warm stratospheric feature near $65-70^{\circ} \mathrm{S}$ latitude is rotating with the neutral atmosphere. This feature may result from a localized wave propagating upward from the troposphere.
\end{abstract}

Key words. radiative transfer - convection - planets and satellites: individual: Neptune

\section{Observations}

Despite the limited solar radiation available to power its atmospheric motions, Neptune has a dynamically active atmosphere. This was evident in visible images taken during the 1989 flyby of Neptune by the Voyager 2 spacecraft (Smith et al. 1989) and in subsequent earth-based imaging (Sromovsky et al. 2002; Max et al. 2003; Feuchtgruber \& Encrenaz 2003). Valuable information on dynamical and radiative processes in Neptune's atmosphere can also be gained from measurements of its temperature field. At the beginning of our study, no observations of Neptune's temperature field had been published since the Voyager 2 IRIS experiment (Conrath et al. 1998) which covered only $80^{\circ} \mathrm{S}-20^{\circ} \mathrm{N}$ latitude, omitting any direct measurements of the south pole during its long summer season. Therefore we acquired spatially resolved thermal images of Neptune in spectral regions sensitive to its upper tropospheric and stratospheric temperatures.

The thermal images shown in Fig. 1 were obtained with the facility mid-infrared camera/spectrometer, VISIR (Lagage et al. 2004), on the Very Large Telescope UT-3 (Melipal) on 1-2 September 2006 (UT). We selected filters to probe near the tropopause $(17.6$ and $18.7 \mu \mathrm{m})$ and in the stratosphere (8.6 and $12.3 \mu \mathrm{m})$. Stratospheric images were recorded twice, allowing us to investigate variability in longitude as Neptune rotated.

\section{Tropopause}

The 17.6- and 18.7- $\mu \mathrm{m}$ filtered radiances are sensitive to a portion of Neptune's spectrum dominated by $\mathrm{H}_{2}$ collision-induced absorption. Because $\mathrm{H}_{2}$ is uniformly mixed in the atmosphere, any observed variability of the 17.6 - and $18.7-\mu \mathrm{m}$ radiance is the result of temperature variations emerging from the 2-200 mbar pressure range, with a much smaller effect arising from variations of the para- vs. ortho- $\mathrm{H}_{2}$ ratio (Conrath et al. 1998). Because the point spread function of the telescope is such a large fraction of Neptune's disk, we deconvolved the images using the Pixon approach (Puetter \& Yahil 1999), with the stellar images shown in Fig. 1 guiding our choice of point spread functions.

Because the contribution functions of upwelling radiance for both of the filters centered at 17.6 and $18.7 \mu \mathrm{m}$ are strongly bifurcated around the level of the temperature minimum and are highly overlapping (Fig. 2), we did not attempt a formal temperature retrieval. Instead, we simply perturbed an a priori temperature structure (Fig. 2, red line) which is consistent both with previous ground-based (Orton et al. 1987, 1990, 1992) and more recent Spitzer (Orton et al. 2005) disk-averaged spectra of Neptune to determine the latitudinal variability of zonal mean temperatures at 100 mbar pressure.

For each of these filters, the temperature profile was changed by the same $\delta T$ at all levels until a match was achieved for the upwelling radiance. At each latitude, these constraining radiances were averaged over the central $14^{\circ}$ of longitude around Neptune's central meridian in cylindrical maps of the calibrated, deconvolved images. This longitude range provides sufficient independent sampling of each latitude, including adequate statistics for the highest northern latitudes shown in the figure. Our initial approach to the absolute calibration for each image was achieved by ratioing the observed flux in each pixel to the total flux of the calibration stars identified in Fig. 1 and multiplying by the appropriate flux for each filter and standard derived by VLT staff scientists for VISIR 


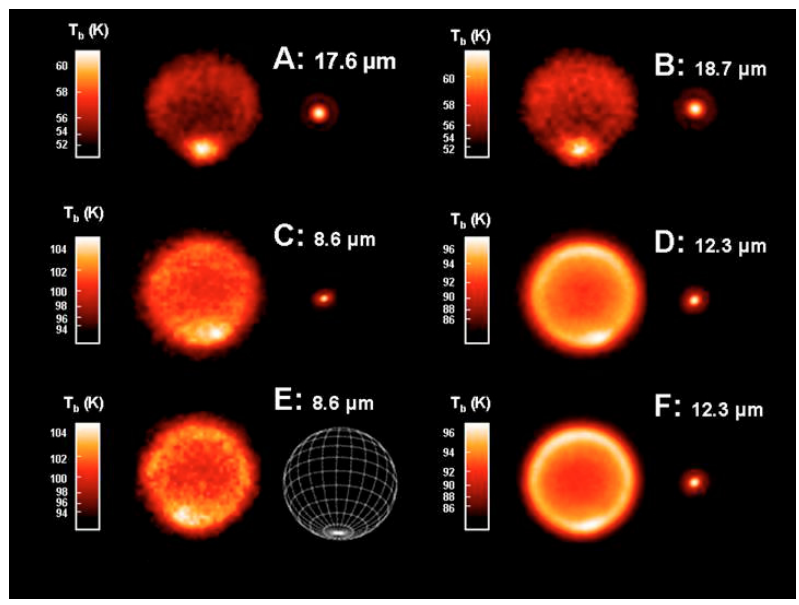

Fig. 1. Thermal images of Neptune obtained in this study, with north at the top of and the south pole visible at the bottom of each image (see the graphic in panel E). The figure also displays images of nearby standard stars which were used initially for absolute radiance calibration radiance and to characterize the point-spread function. Images A and B sample temperatures near Neptune's tropopause. Image A was taken on 2 Sep. 2006 between 1:54 and 3:10 UT using a discrete filter centered at $17.6 \mu \mathrm{m}$ (bandwidth $0.7 \mu \mathrm{m}$ ); the calibration star is HD 25025 . Image B was taken on 1 Sep. 2006 between 1:43 and 3:50 UT using a filter centered at $18.7 \mu \mathrm{m}$ (bandwidth $0.8 \mu \mathrm{m}$ ); its calibration star is also HD 216032. Images C-F, all taken on 2 Sep. 2006, sample emission from Neptune's stratosphere. Image $\mathrm{C}$ was taken using a filter centered at $8.6 \mu \mathrm{m}$ (bandwidth $0.4 \mu \mathrm{m}$ ) between $0: 22$ and 0:55 UT; its calibration star is HD 200914. Image D was taken using a filter centered at $12.3 \mu \mathrm{m}$ (bandwidth $0.2 \mu \mathrm{m}$ ) between 0:59 and 1:25 UT and its calibration star is HD 200514. Image E was taken with the same $8.6 \mu \mathrm{m}$ filter as image $\mathrm{C}$ between 7:16 and 7:40 UT, without a nearby standard star. Image $\mathrm{F}$ was taken with the same $12.3 \mu \mathrm{m}$ filter as image $\mathrm{D}$ between 3:16 and 3:38 UT, and it was calibrated by the same stellar observation. The calibration of image $\mathrm{E}$ was normalized to the same value as image $\mathrm{C}$.

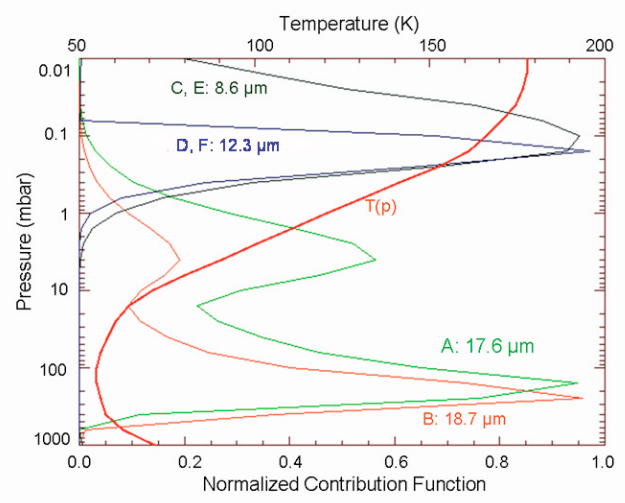

Fig. 2. Contribution functions showing the level-by-level source of the outgoing radiance in the four filters used in the VISIR observations given in Fig. 1. For the 17.6- and $18.7-\mu \mathrm{m}$ emission, the weighting functions peak just below the temperature minimum; the second maxima in the contribution functions near 30-50 mbar are the result of the abrupt rise of the Planck emission with temperature multiplying a weak tail of the weighting function.

and listed in the URL: http://wWw. eso.org/instruments/ visir/tools/zerop_cohen_Jy.txt. However, a comparison with the higher-precision fluxes measured by the IRS instrument on the Spitzer Space Telescope indicated that the $18.7-\mu \mathrm{m}$ radiance should be increased by $34 \%$. This value is not

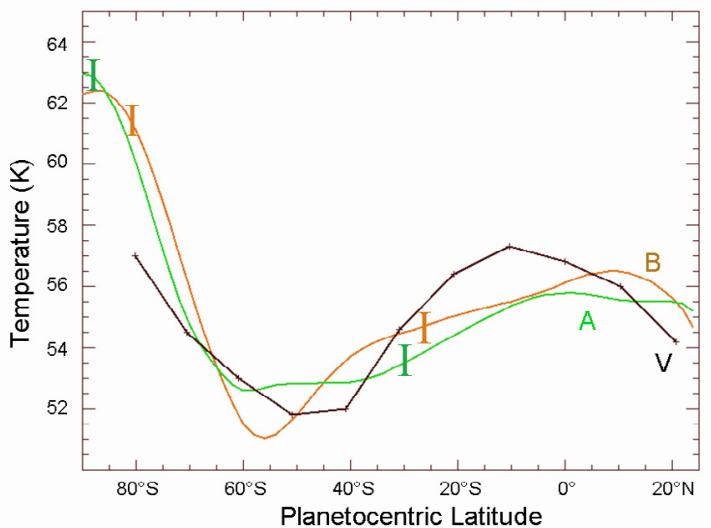

Fig. 3. Latitudinal variations of the zonal-mean temperature at $100 \mathrm{mbar}$ inferred from perturbations of the standard model which match radiances from (A) the 17.6- and (B) the 18.7- $\mu$ m deconvolved images, respectively. Spatial resolution of the deconvolved images is estimated as 0.15 arcsec, or $15000 \mathrm{~km}$. Standard deviations of the zonal mean results are on the order of 1.0-1.5 K, similar to the differences between plots (A) and (B) in the figure. We estimate the uncertainty in the absolute radiance to be $\pm 10 \%$, with its impact on the derived temperatures denoted by the error bars. These are compared with (V) zonal-mean results from the Voyager-2 IRIS experiment (Conrath et al. 1998).

unreasonable, given that the $18.7-\mu \mathrm{m}$ observations on 1 Sep. followed an extremely rare day of torrential rain, and the water vapor in the optical path probably underwent a substantial drop between the Neptune and the standard star observations. The calibrated radiance of the $17.8-\mu \mathrm{m}$ image agrees with the Spitzer spectrum to within $6 \%$, a value well within the uncertainties associated with ground-based radiometry in this spectral region. The Spitzer spectral radiances are nominally considered accurate to better than $10 \%$, a value which we assume as the absolute radiance uncertainty.

Figure 3 displays the 100-mbar temperatures derived independently from the deconvolved 17.6- and 18.7- $\mu$ m images. An additional verification was used to this approach. A model image was devised from the derived temperatures, assuming longitudinal homogeneity (no longitudinal inhomogeneity in the 17.6or $18.7-\mu \mathrm{m}$ images was detected above the noise). This model was then convolved with the point-spread function, based on smoothed versions of the stellar images shown in panels A and B of Fig. 1. The difference between the observed and the model brightness temperatures at a given latitude along Neptune's central meridian was then used to provide a new perturbation to the temperature at that latitude. Except at the latitude extremes, the perturbations were averaged over $6^{\circ}$ of latitude for stability. Two iterations resulted in residuals which were at or below $0.1 \mathrm{~K}$ over most of the planet, with $0.3 \mathrm{~K}$ residuals near the pole.

The latitudinal variation of 100 -mbar temperatures is in qualitative agreement with the Voyager-2 IRIS infrared spectrometer results, exhibiting a clear minimum around $50-60^{\circ} \mathrm{S}$ latitude and a broad maximum around the equator. At the south pole, however, a distinct maximum is detected in both filters of the VISIR data beyond the southern limit of the Voyager 2 IRIS coverage. The south polar temperature is higher than elsewhere on the planet by $7-10 \mathrm{~K}$. The thermal enhancement observed at the south pole is most likely to be the consequence of maximum solar irradiation over several decades, with southern summer solstice having occurred in July 2005. A similar effect was observed on Saturn in 2004 (Orton \& Yanamandra-Fisher 2005). 
The warm south pole provides a mechanism to maintain high $\mathrm{CH}_{4}$ abundances in Neptune's stratosphere. Analyses of Neptune's thermal spectrum (Orton et al. 1987, 1990, 1992, 2005), imply a disk-averaged stratospheric $\mathrm{CH}_{4}$ volume mixing ratio in the range of $0.75-1.5 \times 10^{-3}$, factors of several higher than those determined from saturation vapor pressure (Younglove \& Ely 1987) for the disk-averaged temperature minimum. This can be explained by a strong latitudinal gradient of the $\mathrm{CH}_{4}$ abundance which is in saturation equilibrium with the local temperature minimum. At the south pole, our derived 100 -mbar temperature of $62-66 \mathrm{~K}$ corresponds to a $\mathrm{CH}_{4}$ volume mixing ratio around $8-10 \times 10^{-3}$, i.e. higher by a factor of $\sim 8$ than its observed planetary-mean value. North of $60^{\circ} \mathrm{S}$ latitude, 100 -mbar temperatures range from $\sim 53-57 \mathrm{~K}$, with the corresponding $\mathrm{CH}_{4}$ volume mixing ratio ranging from $3-10 \times 10^{-4}$ - in general lower than the observed mean value. Neptune's stratospheric circulation might also be sufficiently vigorous to transport $\mathrm{CH}_{4}$ away from the pole fast enough that volume mixing ratios in excess of these local equilibrium value could be found. In any case, the high $\mathrm{CH}_{4}$ volume mixing ratio at high southern latitudes may well explain, in part, why relatively high values are reported from thermal spectra where the diskaveraged emission is weighted toward the high-temperature, abundant- $\mathrm{CH}_{4}$ south polar region, whereas other studies at shorter wavelengths using reflected or attenuated sunlight tend to find lower values which are unweighted by those emissions (Baines \& Hammel 1994). We note also that $\mathrm{CH}_{4}$ enhancement at the south pole may also lead to enhanced emissions of other hydrocarbons such as $\mathrm{C}_{2} \mathrm{H}_{2}$ and $\mathrm{C}_{2} \mathrm{H}_{6}$ which are products of $\mathrm{CH}_{4}$ photolysis.

\section{Stratosphere}

At the shorter wavelengths (images $\mathrm{C}-\mathrm{F}$ in Fig. 1), the observed radiances originate in Neptune's stratosphere, as a result of strong molecular opacities superimposed over the weaker $\mathrm{H}_{2}$ continuum. Emission at $8.6 \mu \mathrm{m}$ is dominated by the opacity of $\mathrm{CH}_{4}$ and $\mathrm{CH}_{3} \mathrm{D}$, and it originates from a broad region centered at 0.1 mbar. Emission at $12.3 \mu \mathrm{m}$ is dominated by the opacity of $\mathrm{C}_{2} \mathrm{H}_{6}$, and it originates from a vertically narrower region centered at 0.2 mbar. These images are all characterized by limb brightening, consistent with the increase of temperature with altitude in the stratosphere. This is particularly true of the $12.3-\mu \mathrm{m}$ images (Fig. 1, D and F) of $\mathrm{C}_{2} \mathrm{H}_{6}$ emission, quantitatively consistent with standard models for the vertical distribution of $\mathrm{C}_{2} \mathrm{H}_{6}$ which increases with altitude (Moses et al. 2005). In addition, the region south of $70^{\circ} \mathrm{S}$ shows enhanced emission compared with more northern latitudes. There is no evidence for the lowlatitude warming which was detected by Voyager-2 IRIS spectra of $\mathrm{C}_{2} \mathrm{H}_{2}$ emission (Bezard et al. 1991).

Each of these images also displays a "hot spot", a discrete region of maximum emission near $65-70^{\circ} \mathrm{S}$ which is distinct from the south pole, unlike the emission at 17.6 and $18.7 \mu \mathrm{m}$. The hot spots in the first 8.6- and 12.3- $\mu \mathrm{m}$ (Fig. 1, C and D) images are coincident in planetary position. Because $\mathrm{C}_{2} \mathrm{H}_{6}$ is a byproduct of $\mathrm{CH}_{4}$ photolysis, it is possible that the hot spot is an area of $\sim 70 \%$ enhancement of stratospheric abundances of $\mathrm{CH}_{4}$ and $\mathrm{C}_{2} \mathrm{H}_{6}$, but a simpler explanation is that this is a region with stratospheric temperatures which are warmer than the zonal mean by $\sim 3$ Kelvins. The second $12.3-\mu$ m image (Fig. 1, F), taken $2.25 \mathrm{~h}$ later than the first (Fig. 1, D), shows the hot spot rotating onto Neptune's limb.

On the other hand, the hot spots in the two 8.6- $\mu \mathrm{m}$ images, separated by $6.83 \mathrm{~h}$, appear at two different positions relative to

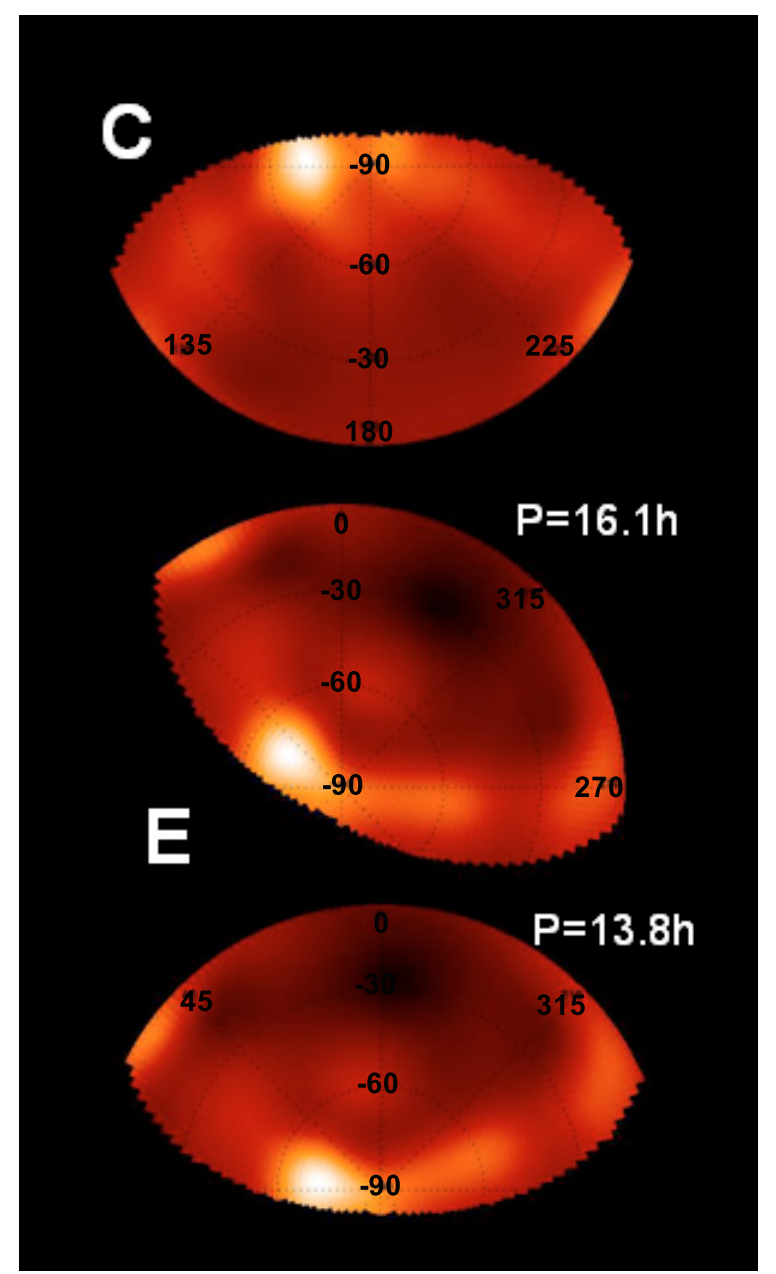

Fig. 4. Polar projection of the deconvolved form of the 8.6- $\mu \mathrm{m}$ images ( $\mathrm{C}$ and $\mathrm{E}$ in Fig. 1), separated by $6.83 \mathrm{~h}$ in time. In the projection of the deconvolved $\mathrm{C}$ image, two different assumptions are made about the longitude system: (1) it rotates with the magnetic rotation period of $16.1 \mathrm{~h}$ (center), and (2) it rotates with a period derived from the bestfit correlation between two two features, $13.8 \pm 1.0 \mathrm{~h}$ (bottom). If the magnetic rotation period is assumed, the bright spot in images $\mathrm{C}$ and $\mathrm{E}$ must be interpreted as two independent regions of enhanced emission in the lower stratosphere. On the other hand, the 13.8-h period is closer ot the $\sim 12-\mathrm{h}$ atmospheric rotation period for latitude $70^{\circ}$.

the central meridian. Figure 4 shows that the features are identical if a rotation period of $13.8 \pm 1.0 \mathrm{~h}$ is assumed. The magnetic rotation period of $16.1 \mathrm{~h}$ (Zarka et al. 1995) requires there to be two features. This argues against the likelihood that this feature is related to auroral processes, which would be associated with the magnetic rotation period. Furthermore, Neptune's magnetic dipole is tilted by about $47^{\circ}$ with respect to its rotational axis (Ness et al. 1995); if the feature observed in the first $8.6 \mu \mathrm{m} \mathrm{im-}$ age (Fig. 1, C) were auroral in origin and coupled to the configuration of the magnetic field, we would expect it to appear $6.83 \mathrm{~h}$ later in image $\mathrm{E}$ at a different latitude. Thus, the feature is more likely to be associated with the atmospheric rotation. The difference between the reported rotation period of $\sim 12.5 \mathrm{~h}$ at $65-70^{\circ} \mathrm{S}$ (Limaye \& Sromovsky 1991) and our value could result from (i) change of the zonal wind speed over time, (ii) difference in the altitude of the winds sampled, or (iii) inability of our images to discriminate more accurately than $5^{\circ}$ of latitude this close to the pole (where there is a steep meridional gradient of the zonal wind profile). 
Our results can be compared to other images of stratospheric emission recorded at the Keck II Telescope on 5 and 6 September, 2003, at 8.0, 8.9 and $11.7 \mu \mathrm{m}$ (Martin et al. 2006) and at the Gemini North telescope on 4 and 5 July, 2005, at 7.7 and $11.7 \mu \mathrm{m}$ (Hammel et al. 2007). A general enhancement of thermal radiance around the south pole was present in all these images, in general agreement with our results. There is no hint in the 2003 Keck results (Martin et al. 2006) of zonal variability, although some longitudinal variability (equivalent to about 1 Kelvin in brightness temperature) is evident near the south pole in the Gemini images in one hemisphere which is correlated in both the 7.7- $\mu \mathrm{m} \mathrm{CH}_{4}$ and 11.7- $\mu \mathrm{m} \mathrm{C}_{2} \mathrm{H}_{6}$ emission (Hammel et al. 2007).

One possible cause of the localized stratospheric heating near $70^{\circ} \mathrm{S}$ is heating by a cometary or asteroidal impactor, similar to the localized heating of Jupiter's stratosphere by the Comet Shoemaker-Levy 9 fragment collisions (Orton et al. 1995). Such a collision would effectively heat the stratosphere but not the troposphere. This explanation is consistent with the suggestion made on the basis of submillimeter $\mathrm{CO}$ observations, namely that oxygen in Neptune's stratosphere is delivered through recent impacts by cometary fragments or Kuiper Belt Objects (Lellouch et al. 2005). We note that the cold temperature of Neptune's lower stratosphere would favor a very slow radiative cooling, as compared to Jupiter, so that the lifetime of stratospheric heating could be significantly longer than in the Shoemaker-Levy 9 collision.

However, the previous detection of some near-polar longitudinal variability of stratospheric emission (Hammel et al. 2007) suggests that the feature has a dynamical origin, although no direct analogue exists in the atmospheres of the other giant planets. Jupiter's low-latitude quasi-quadrennial oscillation (QQO), most likely the result of interactions of small-scale, short-period gravity waves (Leovy et al. 1991), measurably perturbs stratospheric temperatures, but on a global scale. Only in two cases in Jupiter have isolated stratospheric features been detected, both narrow and arc-like in appearance (Orton et al. 1991). Nonetheless, if we hypothesize that an isolated upward-propagating wave was responsible for the $3 \mathrm{~K}$ increase of its 0.1 -mbar temperature, the 100 -mbar perturbation responsible for it would scale to a mere $0.01 \mathrm{~K}$. This is far below the noise levels of the $17.6-\mu \mathrm{m}$ image, where it would otherwise have been detectable near the planetary limb. In fact, this latitude is well known for highly varying cloud activity, including the sudden appearance of broad, singular cloud features (Sromovsky et al. 1993; Rages et al. 2002).

The warm polar temperatures in the troposphere suggest the presence of a vortex which possibly extends into the stratosphere. In some respects, the temperature pattern observed in Neptune's stratosphere resembles that often seen in Earth's winter polar vortex during the peak of a sudden stratospheric warming (Andrews et al. 1995), where a region of relatively high stratospheric temperatures may form, offset from the pole and within a confined range of longitudes. If a similar phenomenon operates on Neptune, however, it would have to do so in the context of the breakdown of a warm summer polar vortex for which there is no terrestrial analog.

\section{Conclusions}

Radiatively-driven elevated temperatures at Neptune's south polar tropopause create an avenue for methane gas transport out of the troposphere where it is effectively segregated by coldtrapping elsewhere on the planet. This should establish a latitudinal distribution of stratospheric $\mathrm{CH}_{4}$ which peaks at the south pole. Its northward gradient will be shallow if the rate of the equatorward transport is more rapid than the rate of downward diffusion. This implies that the seasonally-driven methane leak will be located at its north pole some 80 years from now. The appearance of a discrete high-latitude stratospheric hot spot has no immediate analogue in other planetary atmospheres. Its location near a latitude which has documented large-scale dynamical behavior argues that it is the consequence of an upwelling thermal wave rather than an external event, such as a recent impact. A better understanding of upward propagation of waves in Neptune would most easily ensue from simultaneous observations of cloud structure dynamics and the thermal structure at several levels of the stratosphere. For example adding thermal imaging observations closer to the 7.7- $\mu \mathrm{m} \mathrm{CH}_{4}$ absorption peak, as well as at $8.6 \mu \mathrm{m}$, would determine properties of the disturbance both at the 0.1-mbar level and higher. These observations and their comparison with the more abundant thermal observations of Jupiter and Saturn argue that the atmosphere of Neptune may be the most active of them all, despite its much lower level of solar energy input.

Acknowledgements. We would like to thank K. Baines, M. Burgdorf, B. Fisher, A. Heras, H. Hammel, A. Mainzer, V. Meadows, K. Rages, and L. Sromovsky for useful comments and support. We acknowledge the great help of the European Southern Observatory staff at the Very Large Telescope for making these observations possible. G.S.O. and A.J.F. acknowledge support from grants to the Jet Propulsion Laboratory, California Institute of Technology, from the US National Aeronautics and Space Administration. T.E. acknowledges support from the Centre National de la Recherche Scientifique. C.L. acknowledges support from the NASA Postdoctoral Program. The radiative-transfer calculations were performed on JPL supercomputer facilities which were provided by funding from the JPL Office of the Chief Information Officer.

\section{References}

Andrews, D. G., Holton, J. R., \& Leovy, C. B. 1995, Middle atmosphere dynamics (Academic Press), 489

Baines, K. H., \& Hammel, H. B. 1994, Icarus, 109, 20

Bezard, B., Romani, P. N., Conrath, B. J., \& Maguire, W. C. 1991, J. Geophys. Res., 96, 18961

Conrath, B. J., Gierasch, P. J., \& Ustinov, E. A. 1998, Icarus, 135, 501 Feuchtgruber, H., \& Encrenaz, T. 2003, A\&A, 403, L7

Hammel, H. B., Sitko, M. L., Lynch, D. K., et al. 2007, AJ, 134, 637

Lagage, P. O., Pel, J. W., Authier, M., et al. 2004, The Messenger, 117, 12 Lellouch, E., Moreno, R., \& Paubert, G. 2005, A\&A, 430, L37

Leovy, C. B., Friedson, A. J., \& Orton, G. S. 1991, Nature, 354, 380

Limaye, S. S., \& Sromovsky, L. A. 1991, J. Geophys. Res., 96, 18941

Martin, S., de Pater, I., Kloosterman, J., Gibbard, S., \& Hammel, H. B. 2006, Bull. Am. Astron. Soc., 38, 502

Max, C. E., Macintosh, B. A., Gibbard, S. G., et al. 2003, AJ, 125, 364

Moses, J. I., Fouchet, T., Bézard, B., et al. 2005, J. Geophys. Res. - Planets, 110, 8001

Ness, N. F., Acua, M. H., \& Connerney, J. E. P. 1995, Neptune's magnetic field and field-geometric properties, Neptune and Triton (Tucson: University of Arizona Press), 141

Orton, G., A’Hearn, M., Baines, K., et al. 1995, Science, 267, 1277

Orton, G., Burgdorf, M., Meadows, V., et al. 2005, Bull. Am. Astron. Soc., 37, 662

Orton, G. S., \& Yanamandra-Fisher, P. A. 2005, Science, 307, 696

Orton, G. S., Aitken, D. K., Smith, C., et al. 1987, Icarus, 70,

Orton, G. S., Baines, K. H., Caldwell, J., et al. 1990, Icarus, 85, 257

Orton, G. S., Friedson, A. J., Caldwell, J., et al. 1991, Science, 252, 537

Orton, G. S., Lacy, J. H., Achtermann, J. M., Parmar, P., \& Blass, W. E. 1992, Icarus, 100, 541

Puetter, R. C., \& Yahil, A. 1999, in Astronomical Data Analysis Software and Systems VIII, ed. D. M. Mehringer, R. L. Plante, \& D. A. Roberts, ASP Conf. Ser., 172, 307

Rages, K., Hammel, H. B., \& Lockwood, G. W. 2002, Icarus, 159, 262

Smith, B. A., Soderblom, L. A., Banfield, D., et al. 1989, Science, 246, 1422

Sromovsky, L. A., Fry, P. M., \& Baines, K. H. 2002, Icarus, 156, 16

Sromovsky, L. A., Limaye, S. S., \& Fry, P. M. 1993, Icarus, 105, 140

Younglove, B. A., \& Ely, J. F. 1987, J. Phys. Chem. Ref. Data, 16, 577

Zarka, P., Pedersen, B. M., Lecacheux, A., et al. 1995, Radio emissions from

Neptune, in Neptune and Triton (Tucson: University of Arizona Press), 341 